\title{
Lower Error Bounds for Randomized Multilevel and Changing Dimension Algorithms
}

\author{
Michael Gnewuch
}

\begin{abstract}
We provide lower error bounds for randomized algorithms that approximate integrals of functions depending on an unrestricted or even infinite number of variables. More precisely, we consider the infinite-dimensional integration problem on weighted Hilbert spaces with an underlying anchored decomposition and arbitrary weights. We focus on randomized algorithms and the randomized worst case error. We study two cost models for function evaluation which depend on the number of active variables of the chosen sample points. Multilevel algorithms behave very well with respect to the first cost model, while changing dimension algorithms and also dimension-wise quadrature methods, which are based on a similar idea, can take advantage of the more generous second cost model. We prove the first non-trivial lower error bounds for randomized algorithms in these cost models and demonstrate their quality in the case of product weights. In particular, we show that the randomized changing dimension algorithms provided in [L. Plaskota, G. W. Wasilkowski, J. Complexity 27 (2011), 505-518] achieve convergence rates arbitrarily close to the optimal convergence rate.
\end{abstract}

\section{Introduction}

Integrals over functions with an unbounded or infinite number of variables are important in physics, quantum chemistry or in quantitative finance, see, e.g., [8, 25] and the references therein. In the last few years a large amount of research was dedicated to design new algorithms as, e.g., multilevel and changing dimension algorithms or dimension-wise quadrature methods, to approximate such integrals efficiently. Multilevel algorithms were introduced by Heinrich in [12] in the context of integral equations and by Giles in [8] in the context of stochastic differential equations. Changing dimension algorithms were introduced by Kuo et al. in [16]

Michael Gnewuch, School of Mathematics and Statistics, University of New South Wales, Sydney, NSW, 2052, Australia, e-mail: michael.gnewuch@unsw.edu.au| 
in the context of infinite-dimensional integration on weighted Hilbert spaces and dimension-wise quadrature methods were introduced by Griebel and Holtz in [11] for multivariate integration; changing dimension and dimension-wise quadrature algorithms are based on a similar idea.

Here we want to study the complexity of numerical integration on a weighted Hilbert space of functions with infinitely many variables as it has been done in [14, 16, 13, 18, 9, 21, 2, 4, 6]. The Hilbert space we consider here allows for a so-called anchored function space decomposition. For a motivation of this specific function space setting and connections to problems in stochastics and mathematical finance see, e.g., [13, 18]. We derive lower error bounds for randomized algorithms to solve the infinite-dimensional integration problem. Notice that the complexity of integration problems is less well understood in the randomized setting than in the deterministic setting (where only deterministic algorithms are permitted and the deterministic worst case error is considered), see, e.g., the comments in [20, p. 487].

Our error bounds are for the randomized worst case error and are expressed in terms of the cost of a randomized algorithm. Here we solely take account of function evaluations, i.e., the cost of function sampling, and disregard other cost as, e.g., combinatorial cost. Notice that this makes the statements of our lower bounds only stronger. To evaluate the cost of sampling, we consider two sampling models: the nested subspace sampling model (introduced in [5], where it was called variable subspace sampling model) and the unrestricted subspace sampling model (introduced in [16]). Our lower error bounds are the first non-trivial lower bounds in these settings, cf. also the comments in the introductions of [13, 21]. Due to space restrictions, we do not provide new constructive upper error bounds. For the same reason we refer for a formal definition of multilevel algorithms and changing dimension algorithms for the infinite-dimensional integration problem on weighted Hilbert spaces to [13, 18, 9] and [16, 21], respectively. In this article we only compare our lower bounds to already known upper bounds. In particular, we show that the randomized changing dimension algorithms provided for product weights in [21] achieve convergence rates arbitrarily close to the optimal rate of convergence.

Let us mention that similar general lower error bounds for infinite-dimensional integration on weighted Hilbert spaces are provided in [6] in the determistic setting for the anchored decomposition and in [4] in the randomized setting for underlying ANOVA-type decompositions (to treat the latter decompositions, a technically more involved analysis is necessary).

The article is organized as follows: In Section 2 the setting we want to study is introduced. In Section 3 we prove new lower bounds for the complexity of randomized algorithms for solving the infinite-dimensional integration problem on weighted Hilbert spaces. In Section 3.1 we provide the most general form of our lower bounds which is valid for arbitrary weights. In Section 3.2 we state the simplified form of our lower bounds for specific classes of weights. In particular, we show in Section 3.2.1 that the randomized changing dimension algorithms from [21] are essentially optimal. 


\section{The general setting}

In this section we describe the precise setting we want to study. A comparison with the (slightly different) settings described in the papers [9, 16, 21] will be provided in the forthcoming paper [10]; we refer to the same paper and to [14, 13] for rigorous proofs of the statements on the function spaces we consider here.

\subsection{Notation}

For $n \in \mathbb{N}$ we denote the set $\{1, \ldots, n\}$ by $[n]$. If $u$ is a finite set, then its size is denoted by $|u|$. We put $\mathscr{U}:=\{u \subset \mathbb{N}|| u \mid<\infty\}$. We use the common Landau symbol $O$, and additionally for non-negative functions $f, g:[0, \infty) \rightarrow[0, \infty)$ the notation $f=\Omega(g)$ if $g=O(f)$.

\subsection{The function spaces}

As spaces of integrands of infinitely many variables, we consider reproducing kernel Hilbert spaces; our standard reference for these spaces is [1].

We start with univariate functions. Let $D \subseteq \mathbb{R}$ be a Borel measurable set of $\mathbb{R}$ and let $k: D \times D \rightarrow \mathbb{R}$ be a measurable reproducing kernel with anchor $a \in D$, i.e., $k(a, a)=0$. This implies $k(\cdot, a) \equiv 0$. We assume that $k$ is non-trivial, i.e., $k \neq 0$. We denote the reproducing kernel Hilbert space with kernel $k$ by $H=H(k)$ and its scalar product and norm by $\langle\cdot, \cdot\rangle_{H}$ and $\|\cdot\|_{H}$, respectively. Additionally, we denote its norm unit ball by $B(k)$. We use corresponding notation for other reproducing kernel Hilbert spaces. If $g$ is a constant function in $H$, then the reproducing property implies $g=g(a)=\langle g, k(\cdot, a)\rangle_{H}=0$. Let $\rho$ be a probability measure on $D$. We assume that

$$
M:=\int_{D} k(x, x) \rho(\mathrm{d} x)<\infty .
$$

For arbitrary $\mathbf{x}, \mathbf{y} \in D^{\mathbb{N}}$ and $u \in \mathscr{U}$ we define

$$
k_{u}(\mathbf{x}, \mathbf{y}):=\prod_{j \in u} k\left(x_{j}, y_{j}\right)
$$

where by convention $k_{\emptyset} \equiv 1$. The Hilbert space with reproducing kernel $k_{u}$ will be denoted by $H_{u}=H\left(k_{u}\right)$. Its functions depend only on the coordinates $j \in u$. If it is convenient for us, we identify $H_{u}$ with the space of functions defined on $D^{u}$ determined by the kernel $\prod_{j \in u} k\left(x_{j}, y_{j}\right)$, and write $f_{u}\left(\mathbf{x}_{u}\right)$ instead of $f_{u}(\mathbf{x})$ for $f_{u} \in H_{u}, \mathbf{x} \in D^{\mathbb{N}}$, and $\mathbf{x}_{u}:=\left(x_{j}\right)_{j \in u} \in D^{u}$. For all $f_{u} \in H_{u}$ and $\mathbf{x} \in D^{\mathbb{N}}$ we have

$$
f_{u}(\mathbf{x})=0 \quad \text { if } x_{j}=a \text { for some } j \in u \text {. }
$$


This property yields an anchored decomposition of functions, see, e.g., [17].

Let now $\gamma=\left(\gamma_{u}\right)_{u \in \mathscr{U}}$ be weights, i.e., a family of non-negative numbers. We assume

$$
\sum_{u \in \mathscr{U}} \gamma_{u} M^{|u|}<\infty
$$

Let us define the domain $\mathscr{X}$ of functions of infinitely many variables by

$$
\mathscr{X}:=\left\{\mathbf{x} \in D^{\mathbb{N}} \mid \sum_{u \in \mathscr{U}} \gamma_{u} k_{u}\left(x_{j}, x_{j}\right)<\infty\right\} .
$$

Let $\mu$ be the infinite product probability measure of $\rho$ on $D^{\mathbb{N}}$. Due to our assumptions we have $\mu(\mathscr{X})=1$, see [13, Lemma 1] or [10]. We define

$$
K_{\gamma}(\mathbf{x}, \mathbf{y}):=\sum_{u \in \mathscr{U}} \gamma_{u} k_{u}(\mathbf{x}, \mathbf{y}) \quad \text { for all } \mathbf{x}, \mathbf{y} \in \mathscr{X} .
$$

$K_{\gamma}$ is well-defined and, since $K_{\gamma}$ is symmetric and positive semi-definite, it is a reproducing kernel on $\mathscr{X} \times \mathscr{X}$, see [1]. We denote the corresponding reproducing kernel Hilbert space by $\mathscr{H}_{\gamma}=H\left(K_{\gamma}\right)$, its norm by $\|\cdot\|_{\gamma}$, and its norm unit ball by $B_{\gamma}=B\left(K_{\gamma}\right)$. For the next lemma see [14, Cor. 5] or [10].

Lemma 1. The space $\mathscr{H}_{\gamma}$ consists of all functions $f=\sum_{u \in \mathscr{U}} f_{u}, f_{u} \in H_{u}$, that have a finite norm

$$
\|f\|_{\gamma}=\left(\sum_{u \in \mathscr{U}} \gamma_{u}^{-1}\left\|f_{u}\right\|_{H_{u}}^{2}\right)^{1 / 2} .
$$

For $u \in \mathscr{U}$ let $P_{u}$ denote the orthogonal projection $P_{u}: \mathscr{H}_{\gamma} \rightarrow H_{u}, f \mapsto f_{u}$ onto $H_{u}$. Then each $f \in \mathscr{H}_{\gamma}$ has a unique representation

$$
f=\sum_{u \in \mathscr{U}} f_{u} \quad \text { with } f_{u}=P_{u}(f) \in H_{u}, u \in \mathscr{U} .
$$

\subsection{Infinite-dimensional integration}

For a given $f \in \mathscr{H}_{\gamma}$ we want to approximate the integral

$$
I(f):=\int_{\mathscr{X}} f(\mathbf{x}) \mu(\mathrm{d} \mathbf{x}) .
$$

Due to $\left[3, I\right.$ is continuous on $\mathscr{H}_{\gamma}$ and its representer $h \in \mathscr{H}_{\gamma}$ is given by

$$
h(\mathbf{x})=\int_{\mathscr{X}} K_{\gamma}(\mathbf{x}, \mathbf{y}) \mu(\mathrm{d} \mathbf{y}) .
$$

The operator norm of the integration functional $I$ is given by 


$$
\|I\|_{\mathscr{H}}^{2}=\|h\|_{\gamma}^{2}=\sum_{u \in \mathscr{U}} \gamma_{u} C_{0}^{|u|}<\infty
$$

where

$$
C_{0}:=\int_{D} \int_{D} k(x, y) \rho(\mathrm{d} x) \rho(\mathrm{d} y) .
$$

We have $C_{0} \leq M$. We assume that $I$ is non-trivial, i.e., that $C_{0}>0$ and $\gamma_{u}>0$ for at least one $u \in \mathscr{U}$. For $u \in \mathscr{U}$ and $f \in \mathscr{H}_{\gamma}$ we define $I_{u}:=I \circ P_{u}$, i.e.,

$$
I_{u}(f)=\int_{D^{u}} f_{u}\left(\mathbf{x}_{u}\right) \rho^{u}\left(\mathrm{~d} \mathbf{x}_{u}\right),
$$

and the representer $h_{u}$ of $I_{u}$ in $\mathscr{H}_{\gamma}$ is given by $h_{u}(\mathbf{x})=P_{u}(h)(\mathbf{x})$. Thus we have

$$
h=\sum_{u \in \mathscr{U}} h_{u} \quad \text { and } \quad I(f)=\sum_{u \in \mathscr{U}} I_{u}\left(f_{u}\right) \quad \text { for all } f \in \mathscr{H}_{\gamma} .
$$

Furthermore,

$$
\left\|h_{u}\right\|_{\gamma}^{2}=\gamma_{u} C_{0}^{|u|} \text { for all } u \in \mathscr{U} .
$$

\subsection{Randomized algorithms, cost models, and errors}

As in [13], we assume that algorithms for approximation of $I(f)$ have access to the function $f$ via a subroutine ("oracle") that provides values $f(\mathbf{x})$ for points $\mathbf{x} \in D^{\mathbb{N}}$. For convenience we define $f(\mathbf{x})=0$ for $\mathbf{x} \in D^{\mathbb{N}} \backslash \mathscr{X}$.

We now present the cost models introduced in [5] and [16]: In both models we only consider the cost of function evaluations. To define the cost of a function evaluation, we fix a monotone increasing function $\$(v): \mathbb{N}_{0} \rightarrow[1, \infty]$. For our lower error bounds we will later assume that $\$(v)=\Omega\left(v^{s}\right)$ for some $s \geq 0$. For each $v \in \mathscr{U}$ we define the finite-dimensional affine subspace $\mathscr{X}_{v, a}$ of $\mathscr{X}$ by

$$
\mathscr{X}_{v, a}:=\left\{\mathbf{x} \in D^{\mathbb{N}} \mid x_{j}=a \text { for all } j \in \mathbb{N} \backslash v\right\} .
$$

In the nested subspace sampling model function evaluations can be done in a sequence of affine subspaces $\mathscr{X}_{v_{1}, a} \subset \mathscr{X}_{v_{2}, a} \subset \cdots$ for a strictly increasing sequence $\mathrm{v}=\left(v_{i}\right)_{i \in \mathbb{N}}$ of sets $\emptyset \neq v_{i} \in \mathscr{U}$, and the cost for each function evaluation is given by the cost function

$$
c_{\mathrm{v}, a}(\mathbf{x}):=\inf \left\{\$\left(\left|v_{i}\right|\right) \mid \mathbf{x} \in \mathscr{X}_{v_{i}, a}\right\},
$$

where we use the standard convention that $\inf \emptyset=\infty$. Let $C_{\text {nest }}$ denote the set of all cost functions of the form (6). The nested subspace sampling model was introduced in [5], where it was actually called "variable subspace sampling model". We prefer the name "nested subspace sampling model" to clearly distinguish this model from the following cost model, which is even more "variable": 
In the unrestricted subspace sampling model we are allowed to sample in any subspace $\mathscr{X}_{u, a}, u \in \mathscr{U}$, without any restriction. The cost for each function evaluation is given by the cost function

$$
c_{a}(\mathbf{x}):=\inf \left\{\$(|u|) \mid \mathbf{x} \in \mathscr{X}_{u, a}, u \in \mathscr{U}\right\} .
$$

Put $C_{\mathrm{unr}}:=\left\{c_{a}\right\}$. The unrestricted subspace sampling model was introduced in [16], where it did not get a particular name. Obviously, the unrestricted subspace sampling model is more generous than the nested subspace sampling model.

We consider randomized algorithms for integration of functions $f \in \mathscr{H}_{\gamma}$. For a formal definition we refer to [5, 19, 23, 24]. Here we require that a randomized algorithm $Q$ yields for each $f \in \mathscr{H}_{\gamma}$ a square-integrable random variable $Q(f)$. (More precisely, a randomized algorithm $Q$ is a map $Q: \Omega \times \mathscr{H}_{\gamma} \rightarrow \mathbb{R},(\omega, f) \mapsto Q(\omega, f)$, where $\Omega$ is some suitable probability space. But for convenience we will not specify the underlying probability space $\Omega$ and suppress any reference to $\Omega$ or $\omega \in \Omega$. We use this convention also for other random variables.) Furthermore, we require that the cost of a randomized algorithm $Q$, which is defined to be the sum of the cost of all function evaluations, is a random variable, which may depend on the function $f$. That is why we denote this random variable by $\operatorname{cost}_{c}(Q, f), c$ the relevant cost function from $C_{\text {nest }}$ or $C_{\mathrm{unr}}$.

We denote the class of all randomized algorithms for numerical integration on $\mathscr{H}_{\gamma}$ that satisfy the very mild requirements stated above by $\mathscr{A}^{\text {ran }}$. For unrestricted subspace sampling we additionally consider a subclass $\mathscr{A}^{\text {res }}$ of $\mathscr{A}^{\text {ran }}$. We say that an algorithm $Q \in \mathscr{A}^{\text {ran }}$ is in $\mathscr{A}^{\text {res }}$ if there exist an $n \in \mathbb{N}_{0}$ and sets $v_{1}, \ldots, v_{n} \in \mathscr{U}$ such that for every $f \in \mathscr{H}_{\gamma}$ the algorithms $Q$ performs exactly $n$ function evaluations of $f$, where the $i$ th sample point is taken from $\mathscr{X}_{v_{i}, a}$, and $\mathbb{E}\left(\operatorname{cost}_{c_{a}}(Q, f)\right)=\sum_{i=1}^{n} \$\left(\left|v_{i}\right|\right)$. If additionally $\left|v_{1}\right|, \ldots,\left|v_{n}\right| \leq \omega$ for some $\omega \in \mathbb{N}$, we say that $Q \in \mathscr{A}^{\text {res }-\omega}$. Notice that the classes $\mathscr{A}^{\text {ran }}, \mathscr{A}^{\text {res }}$, and $\mathscr{A}^{\text {res- } \omega}$ contain in particular non-linear and adaptive algorithms.

The worst case cost of a randomized algorithm $Q$ on a class of integrands $F$ is

$$
\operatorname{cost}_{\text {nest }}(Q, F):=\inf _{c \in C_{\text {nest }}} \sup _{f \in F} \mathbb{E}\left(\operatorname{cost}_{c}(Q, f)\right)
$$

in the nested subspace sampling model and

$$
\operatorname{cost}_{\mathrm{unr}}(Q, F):=\sup _{f \in F} \mathbb{E}\left(\operatorname{cost}_{c_{a}}(Q, f)\right)
$$

in the unrestricted subspace sampling model. The randomized (worst case) error $e(Q, F)$ of approximating the integration functional $I$ by $Q$ on $F$ is defined as

$$
e(Q, F):=\left(\sup _{f \in F} \mathbb{E}\left((I(f)-Q(f))^{2}\right)\right)^{1 / 2} .
$$

For $N \in \mathbb{R}, \bmod \in\{$ nest, unr $\}$, and $* \in\{$ ran, res, res $-\omega\}$ let us define the corresponding Nth minimal error by 


$$
e_{\text {mod }}^{*}(N, F):=\inf \left\{e(Q, F) \mid Q \in \mathscr{A}^{*} \text { and } \operatorname{cost}_{\bmod }(Q, F) \leq N\right\} .
$$

\subsection{Strong Polynomial tractability}

Let $\omega \in \mathbb{N}, \bmod \in\{$ nest, unr $\}$, and $* \in\{$ ran, res, res $-\omega\}$. The $\varepsilon$-complexity of the infinite-dimensional integration problem $I$ on $\mathscr{H}_{\gamma}$ in the considered cost model with respect to the class of admissable randomized algorithms $\mathscr{A}^{*}$ is the minimal cost among all admissable algorithms, whose randomized errors are at most $\varepsilon$, i.e.,

$$
\operatorname{comp}_{\text {mod }}^{*}\left(\varepsilon, B_{\gamma}\right):=\inf \left\{\operatorname{cost}_{\text {mod }}\left(Q, B_{\gamma}\right) \mid Q \in \mathscr{A}^{*} \text { and } e\left(Q, B_{\gamma}\right) \leq \varepsilon\right\} .
$$

The integration problem $I$ is said to be strongly polynomially tractable if there are non-negative constants $C$ and $p$ such that

$$
\operatorname{comp}_{\bmod }^{*}\left(\varepsilon, B_{\gamma}\right) \leq C \varepsilon^{-p} \quad \text { for all } \varepsilon>0 .
$$

The exponent of strong polynomial tractability is given by

$$
p_{\text {mod }}^{*}=p_{\text {mod }}^{*}(\gamma):=\inf \{p \mid p \text { satisfies (9) for some } C>0\} .
$$

Essentially, $1 / p_{\text {mod }}^{*}$ is the convergence rate of the $N$ th minimal error $e_{\bmod }^{*}\left(N, B_{\gamma}\right)$. In particular, we have for all $p>p_{\text {mod }}^{*}$ that $e_{\text {mod }}^{*}\left(N, B_{\gamma}\right)=O\left(N^{-1 / p}\right)$.

\section{Lower Bounds}

We start in Section 3.1 by proving lower bounds for general weights. In Section 3.2 we show how these bounds simplify for several specific classes of weights.

\subsection{Results for General Weights}

Let $\gamma=\left(\gamma_{u}\right)_{u \in \mathscr{U}}$ be a given family of weights that satisfy (3). We denote by $\widehat{\gamma}$ the family of weights defined by

$$
\widehat{\gamma}_{u}:=\gamma_{u} C_{0}^{|u|} \text { for all } u \in \mathscr{U} \text {. }
$$

Recall that (3) implies $\sum_{u \in \mathscr{U}} \widehat{\gamma}_{u}<\infty$. Weights $\gamma$ are called finite-order weights of $\operatorname{order} \omega$ if there exists an $\omega \in \mathbb{N}$ such that $\gamma_{u}=0$ for all $u \in \mathscr{U}$ with $|u|>\omega$. Finiteorder weights were introduced in [7] for spaces of functions with a finite number of variables. The following definition is taken from [9].

Definition 1. For weights $\gamma$ and $\sigma \in \mathbb{N}$ let us define the cut-off weights of order $\sigma$ 


$$
\gamma^{(\sigma)}=\left(\gamma_{u}^{(\sigma)}\right)_{u \in \mathscr{U}} \quad \text { via } \quad \gamma_{u}^{(\sigma)}= \begin{cases}\gamma_{u} & \text { if }|u| \leq \sigma \\ 0 & \text { otherwise }\end{cases}
$$

Cut-off weights of order $\sigma$ are in particular finite-order weights of order $\sigma$. Let us denote by $u_{1}(\sigma), u_{2}(\sigma), \ldots$, the distinct non-empty sets $u \in \mathscr{U}$ with $\gamma_{u}^{(\sigma)}>0$ for which $\widehat{\gamma}_{u_{1}(\sigma)}^{(\sigma)} \geq \widehat{\gamma}_{u_{2}(\sigma)}^{(\sigma)} \geq \cdots$. Let us put $u_{0}(\sigma):=\emptyset$. We can make the same definitions for $\sigma=\infty$; then we have obviously $\gamma^{(\infty)}=\gamma$. For convenience we will often suppress any reference to $\sigma$ in the case where $\sigma=\infty$. For $\sigma \in \mathbb{N} \cup\{\infty\}$ let us define

$$
\operatorname{decay}_{\gamma, \sigma}:=\sup \left\{p \in \mathbb{R} \mid \lim _{j \rightarrow \infty} \widehat{\gamma}_{u_{j}(\sigma)}^{(\sigma)} j^{p}=0\right\} \text {. }
$$

Due to assumption (3) the weights we consider always satisfy decay ${ }_{\gamma, \sigma} \geq 1$ for all $\sigma \in \mathbb{N} \cup\{\infty\}$. The following definition is from [9].

Definition 2. For $\sigma \in \mathbb{N} \cup\{\infty\}$ let $t_{\sigma}^{*} \in[0, \infty]$ be defined as

$$
t_{\sigma}^{*}:=\inf \left\{t \geq\left. 0\left|\exists C_{t}>0 \forall v \in \mathscr{U}:\right|\left\{i \in \mathbb{N} \mid u_{i}(\sigma) \subseteq v\right\}\left|\leq C_{t}\right| v\right|^{t}\right\} .
$$

Let $\sigma \in \mathbb{N}$. Since $\left|u_{i}(\sigma)\right| \leq \sigma$ for all $i \in \mathbb{N}$, we have obviously $t_{\sigma}^{*} \leq \sigma$. On the other hand, if we have an infinite sequence $\left(u_{j}(\sigma)\right)_{j \in \mathbb{N}}$, it is not hard to verify that $t_{\sigma}^{*} \geq 1$, see [9].

For $v_{1}, \ldots, v_{n} \in \mathscr{U}$ we use the short hand $\left\{v_{i}\right\}$ for $\left(v_{i}\right)_{i=1}^{n}$. Put $v:=\cup_{i=1}^{n} v_{i}$ and define the mapping

$$
\Psi_{\left\{v_{i}\right\}, a}:=\sum_{j ; \exists i \in[n]: u_{j} \subseteq v_{i}} P_{u_{j}} .
$$

$\Psi_{\left\{v_{i}\right\}, a}$ is the orthogonal projection of $\mathscr{H}_{\gamma}$ onto the subspace

$$
H_{\left\{v_{i}\right\}, a}:=\sum_{j ; \exists i \in[n]: u_{j} \subseteq v_{i}} H_{u_{j}} .
$$

Put

$$
\mathrm{b}_{\left\{v_{i}\right\}, a}:=\sup _{f \in B_{\gamma}}\left|I(f)-I\left(\Psi_{\left\{v_{i}\right\}, a} f\right)\right| .
$$

In the case where $n=1$ and $v=v_{1}$, we simply write $\Psi_{v, a}$ and $\mathrm{b}_{v, a}$. In that case we have, due to (2),

$$
\left(\Psi_{v, a}(f)\right)(\mathbf{x})=f\left(\mathbf{x}_{v} ; \mathbf{a}\right) \quad \text { for all } f \in \mathscr{H}_{\gamma} \text { and } \mathbf{x} \in \mathscr{X},
$$

where the $j$ th component of $\left(\mathbf{x}_{v} ; \mathbf{a}\right)$ is defined by

$$
\left(\mathbf{x}_{v} ; \mathbf{a}\right)_{j}:= \begin{cases}x_{j} & \text { if } j \in v \\ a & \text { otherwise. }\end{cases}
$$

Lemma 2. Let $v_{1}, \ldots, v_{n} \in \mathscr{U}$. Then 


$$
\mathrm{b}_{\left\{v_{i}\right\}, a}^{2}=\sum_{j ; \forall i \in[n]: u_{j} \nsubseteq v_{i}} \widehat{\gamma}_{u_{j}} .
$$

Proof. Let $h_{\left\{v_{i}\right\}, a}$ denote the representer of the continuous functional $I \circ \Psi_{\left\{v_{i}\right\}, a}$. Due to (12) we get

$$
h_{\left\{v_{i}\right\}, a}=\sum_{j ; \exists i \in[n]: u_{j} \subseteq v_{i}} h_{u_{j}} .
$$

Since $h-h_{\left\{v_{i}\right\}, a}$ is the representer of $I-I \circ \Psi_{\left\{v_{i}\right\}, a}$ in $\mathscr{H}_{\gamma}$, we obtain with (5)

$$
\mathrm{b}_{\left\{v_{i}\right\}, a}^{2}=\left\|h-h_{\left\{v_{i}\right\}, a}\right\|_{\gamma}^{2}=\left\|\sum_{j ; \forall i \in[n]: u_{j} \nsubseteq v_{i}} h_{u_{j}}\right\|_{\gamma}^{2}=\sum_{j ; \forall i \in[n]: u_{j} \nsubseteq v_{i}}\left\|h_{u_{j}}\right\|_{\gamma}^{2}=\sum_{j ; \forall i \in[n]: u_{j} \nsubseteq v_{i}} \widehat{\gamma}_{u_{j}} .
$$

Lemma 3. Let $\theta \in(1 / 2,1]$ and $v_{1}, \ldots, v_{n} \in \mathscr{U}$. Let the randomized algorithm $Q \in$ $\mathscr{A}^{\mathrm{ran}}$ satisfy $\mathbb{P}\left(Q(f)=Q\left(\Psi_{\left\{v_{i}\right\}, a} f\right)\right) \geq \theta$ for all $f \in B_{\gamma}$. Then

$$
e\left(Q, B_{\gamma}\right)^{2} \geq(2 \theta-1) \mathrm{b}_{\left\{v_{i}\right\}, a}^{2} .
$$

Proof. Since $\Psi_{\left\{v_{i}\right\}, a}$ is an orthogonal projection, we have for all $f \in B_{\gamma}$ that $g:=$ $f-\Psi_{\left\{v_{i}\right\}, a} f \in B_{\gamma}$. Furthermore, $\Psi_{\left\{v_{i}\right\}, a}(g)=\Psi_{\left\{v_{i}\right\}, a}(-g)=0$. Let $A:=\{Q(g)=$ $Q(-g)\}$. Then $\left\{Q(g)=Q\left(\Psi_{\left\{v_{i}\right\}, a} g\right)\right\} \cap\left\{Q(-g)=Q\left(\Psi_{\left\{v_{i}\right\}, a}(-g)\right)\right\} \subseteq A$, and hence $\mathbb{P}(A) \geq 2 \theta-1$. Therefore

$$
\begin{aligned}
e\left(Q, B_{\gamma}\right)^{2} & \left.\geq \max \left\{\mathbb{E}\left((I(g)-Q(g))^{2}\right), \mathbb{E}((I(-g)-Q(-g)))^{2}\right)\right\} \\
& \left.\geq \max \left\{\int_{A}(I(g)-Q(g))^{2} \mathrm{~d} \mathbb{P}, \int_{A}(I(-g)-Q(-g))\right)^{2} \mathrm{~d} \mathbb{P}\right\} \\
& \geq(2 \theta-1)|I(g)|^{2}=(2 \theta-1)\left|I(f)-I\left(\Psi_{\left\{v_{i}\right\}, a} f\right)\right|^{2} .
\end{aligned}
$$

Hence $e\left(Q, B_{\gamma}\right)^{2} \geq(2 \theta-1) \sup _{f \in B_{\gamma}}\left|I(f)-I\left(\Psi_{\left\{v_{i}\right\}, a} f\right)\right|^{2}=(2 \theta-1) \mathrm{b}_{\left\{v_{i}\right\}, a}^{2}$.

Further Assumptions. We assume for the rest of this article that $\$(v)=\Omega\left(v^{s}\right)$ for some $s \in(0, \infty)$. Furthermore, we assume that $\gamma_{\{1\}}>0$ and that there exists an $\alpha>0$ such that for univariate integration in $H\left(\gamma_{\{1\}} k\right)$ the $N$ th minimal error satisfies

$$
e^{\mathrm{ran}}\left(N, B\left(\gamma_{\{1\}} k\right)\right)=\Omega\left(N^{-\alpha / 2}\right) .
$$

(Note that in the univariate situation the nested and the unrestricted subspace sampling models are equal; that is why we suppress the reference to unr or nest.) Since $B\left(\gamma_{\{1\}} k\right) \subseteq B$, assumption (14) implies in particular

$$
e_{\text {nest }}^{\text {ran }}\left(N, B_{\gamma}\right)=\Omega\left(N^{-\alpha / 2}\right) \quad \text { and } \quad e_{\mathrm{unr}}^{\text {res- } \omega}\left(N, B_{\gamma}\right) \geq e_{\mathrm{unr}}^{\mathrm{res}}\left(N, B_{\gamma}\right)=\Omega\left(N^{-\alpha / 2}\right) .
$$

Theorem 1. Consider the nested subspace sampling model. To achieve strong polynomial tractability for the class $\mathscr{A}^{\mathrm{ran}}$ it is necessary that the weights satisfy 


$$
\operatorname{decay}_{\gamma, \sigma}>1 \text { for all } \sigma \in \mathbb{N} \text {. }
$$

If (16) holds, we have

$$
p_{\text {nest }}^{\text {ran }} \geq \max \left\{\frac{2}{\alpha}, \sup _{\sigma \in \mathbb{N}} \frac{2 s / t_{\sigma}^{*}}{\operatorname{decay~}_{\gamma, \sigma}-1}\right\} .
$$

As we will see in Section 3.2 for product weights and finite-order weights condition (16) is equivalent to decay $\gamma=\operatorname{decay}_{\gamma, \infty}>1$.

Proof. Let $Q \in \mathscr{A}^{\text {ran }}$ with $\operatorname{cost}_{\text {nest }}\left(Q, B_{\gamma}\right) \leq N$. Then there exists an increasing sequence $\mathrm{v}=\left(v_{i}\right)_{i \in \mathbb{N}}, \emptyset \neq v_{i} \in \mathscr{U}$, such that $\mathbb{E}\left(\operatorname{cost}_{c_{v}, a}(Q, f)\right) \leq N+1$ for every $f \in B_{\gamma}$. Let $m$ be the largest integer satisfying $\$\left(\left|v_{m}\right|\right) \leq 4(N+1)$. This implies for all $f \in B_{\gamma}$ that $\mathbb{P}\left(Q(f)=Q\left(\Psi_{v_{m}, a} f\right)\right) \geq 3 / 4$, see (13). Due to Lemma 2 and 3 we get

$$
e\left(Q, B_{\gamma}\right)^{2} \geq \frac{1}{2} \sum_{j ; u_{j} \notin v_{m}} \widehat{\gamma}_{u_{j}} .
$$

Let us now assume that $\gamma$ are weights of finite order $\omega$. Then we get for $t>t_{\omega}^{*}$ and a suitable constant $C_{t}>0$

$$
\tau_{m}:=\left|\left\{j \mid u_{j} \subseteq v_{m}\right\}\right| \leq C_{t}\left|v_{m}\right|^{t}=O\left(N^{t / s}\right),
$$

since $N=\Omega\left(\left|v_{m}\right|^{s}\right)$. Hence we get for $p_{\omega}>\operatorname{decay}_{\gamma, \omega}=\operatorname{decay}_{\gamma} \geq 1$

$$
e\left(Q, B_{\gamma}\right)^{2} \geq \frac{1}{2} \sum_{j=\tau_{m}+1}^{\infty} \widehat{\gamma}_{u_{j}}=\Omega\left(\tau_{m}^{1-p_{\omega}}\right)=\Omega\left(N^{\frac{t}{s}\left(1-p_{\omega}\right)}\right) .
$$

For general weights $\gamma, \sigma \in \mathbb{N}$, and cut-off weights $\gamma^{(\sigma)}$ we have $e\left(Q, B_{\gamma}\right) \geq e\left(Q, B_{\gamma^{(\sigma)}}\right)$, see also [9, Remark 3.3]. Since the cut-off weights $\gamma^{(\sigma)}$ are weights of finite order $\sigma$, we get for all $p_{\sigma}>$ decay $_{\gamma, \sigma}$ and $t_{\sigma}>t_{\sigma}^{*}$

$$
e\left(Q, B_{\gamma}\right)^{2}=\Omega\left(N^{\frac{t \sigma}{s}\left(1-p_{\sigma}\right)}\right) .
$$

Since (15) holds, the inequality for the exponent of tractability follows.

Now assume that the infinite-dimensional integration problem $I$ is strongly polynomially tractable. Let $\sigma \in \mathbb{N}$. Then we get from inequality (17) that $p_{\sigma} \geq$ $1+2 s /\left(t_{\sigma} p_{\text {nest }}^{\text {ran }}\right)$. Hence

$$
\operatorname{decay}_{\gamma, \sigma} \geq 1+\frac{2 s / t_{\sigma}^{*}}{p_{\text {nest }}^{\text {ran }}} .
$$

Thus we have decay ${ }_{\gamma, \sigma}>1$ for all $\sigma \in \mathbb{N}$.

Theorem 2. Consider the unrestricted subspace sampling model. To achieve strong polynomial tractability for the class $\mathscr{A}^{\mathrm{res}}$ it is necessary that the weights satisfy

$$
\operatorname{decay}_{\gamma, \sigma}>1 \text { for all } \sigma \in \mathbb{N} \text {. }
$$


If this is the case, we have

$$
p_{\text {unr }}^{\mathrm{res}} \geq \max \left\{\frac{2}{\alpha}, \sup _{\sigma \in \mathbb{N}} \frac{2 \min \left\{1, s / t_{\sigma}^{*}\right\}}{\operatorname{decay}_{\gamma, \sigma}-1}\right\} .
$$

Proof. Let $Q \in \mathscr{A}^{\text {res }}$ have cost ${ }_{\text {unr }}\left(Q, B_{\gamma}\right) \leq N$. Then there exists an $n \in \mathbb{N}$ and coordinate sets $v_{1}, \ldots, v_{n}$ such that $Q$ selects randomly $n$ sample points $\mathbf{x}_{1} \in \mathscr{X}_{v_{1}, a}, \ldots, \mathbf{x}_{n} \in$ $\mathscr{X}_{v_{n}, a}$ and $\sum_{i=1}^{n} \$\left(\left|v_{i}\right|\right) \leq N$. Since $Q(f)=Q\left(\Psi_{\left\{v_{i}\right\}, a} f\right)$ for all $f \in B_{\gamma}$, we obtain from Lemma2 and 3

$$
e\left(Q, B_{\gamma}\right)^{2} \geq \sum_{j ; \forall i \in[n]: u_{j} \nsubseteq v_{i}} \widehat{\gamma}_{u_{j}} .
$$

Let us first assume that $\gamma$ are weights of finite order $\omega$. Then we get with Jensen's inequality for $t>t_{\omega}^{*}$ and suitable constants $C_{t}, c>0$

$$
\begin{aligned}
\left|\left\{j \mid \exists i \in[n]: u_{j} \subseteq v_{i}\right\}\right| & \leq \sum_{i=1}^{n}\left|\left\{j \mid u_{j} \subseteq v_{i}\right\}\right| \leq \sum_{i=1}^{n} C_{t}\left|v_{i}\right|^{t} \\
& \leq C_{t}\left(\sum_{i=1}^{n}\left|v_{i}\right|^{s}\right)^{1 / \min \{1, s / t\}} \leq C_{t}(c N)^{1 / \min \{1, s / t\}} .
\end{aligned}
$$

Hence we obtain for $S:=\left\lceil C_{t}(c N)^{1 / \min \{1, s / t\}}\right\rceil$ and all $p_{\omega}>\operatorname{decay}_{\gamma, \omega}$

$$
e\left(Q, B_{\gamma}\right)^{2} \geq \sum_{j=S+1}^{\infty} \widehat{\gamma}_{u_{j}}=\Omega\left(S^{1-p_{\omega}}\right)=\Omega\left(N^{\frac{1-p_{\omega}}{\min \{1, s / t\}}}\right) .
$$

If we have general weights $\gamma$, then we obtain for $\sigma \in \mathbb{N}$ and the cut-off weights $\gamma(\sigma)$ that $e\left(Q, B\left(K_{\gamma}\right)\right) \geq e\left(Q, B\left(K_{\gamma(\sigma)}\right)\right)$. From this and (15) the inequality for the exponent of tractability follows. Similarly as in the proof of Theorem 1 the necessity of condition (16) is easily established.

Theorem 3. Let $\omega \in \mathbb{N}$ be fixed. We have for the exponent of tractability $p_{\mathrm{unr}}^{\mathrm{res}-\omega}$ in the unrestricted subspace sampling setting

$$
p_{\text {unr }}^{\mathrm{res}-\omega} \geq \max \left\{\frac{2}{\alpha}, \sup _{\sigma \in \mathbb{N}} \frac{2}{\operatorname{decay}_{\gamma, \sigma}-1}\right\} .
$$

Proof. We follow the lines of the proof of Theorem 2 and use the same notation. The difference is that this time $Q$ selects randomly $n$ sample points $\mathbf{x}_{1} \in$ $\mathscr{X}_{v_{1}, a}, \ldots, \mathbf{x}_{n} \in \mathscr{X}_{v_{n}, a}$, where $\left|v_{i}\right| \leq \omega$ for all $i \in[n]$, and that we therefore can make the estimate $\left|\left\{j \mid \exists i \in[n]: u_{j} \subseteq v_{i}\right\}\right| \leq 2{ }^{\omega} n=O(N)$, since $\$\left(\left|v_{i}\right|\right) \geq 1$ for all $i \in[n]$ by definition of the function $\$$. Hence we get this time for $p>$ decay $_{\gamma}$

$$
e\left(Q, B_{\gamma}\right)^{2} \geq \sum_{j=2^{\omega_{n+1}}}^{\infty} \widehat{\gamma}_{u_{j}}=\Omega\left(N^{1-p}\right) .
$$


A comparison of Theorem 2 and 3 indicates that there are cost functions and classes of finite-order weights for which changing dimension algorithms cannot achieve convergence rates that are arbitrarily close to the optimal rate. Let us recall that for weights of finite order $\omega$, changing dimension algorithms as defined in [16, Proof of Thm. 5] would only use sample points from sample spaces $\mathscr{X}_{u, a}$ with $|u| \leq \omega$; see also the comment at the beginning of Section 4 in [16]. Examples of such cost functions and finite-order weights would be $\$(k)=\Omega\left(k^{s}\right)$ and lexicographically-ordered weights of order $\omega>s$, see Section 3.2.3 (A similar observation was made for the deterministic setting, see [9, Thm. 3.2 \& Sect. 3.2.3 ].)

\subsection{Results for Specific Classes of Weights}

Here we consider some example classes of weights and show how our bounds from Section 3.1 simplify in those settings.

\subsubsection{Product weights and finite-product weights}

Definition 3. Let $\left(\gamma_{j}\right)_{j \in \mathbb{N}}$ be a sequence of non-negative real numbers satisfying $\gamma_{1} \geq \gamma_{2} \geq \ldots$. With the help of this sequence we define for $\omega \in \mathbb{N} \cup\{\infty\}$ weights $\gamma=\left(\gamma_{u}\right)_{u \in \mathscr{U}}$ by

$$
\gamma_{u}= \begin{cases}\prod_{j \in u} \gamma_{j} & \text { if }|u| \leq \omega, \\ 0 & \text { otherwise }\end{cases}
$$

where we use the convention that the empty product is 1 . In the case where $\omega=\infty$, we call such weights product weights, in the case where $\omega$ is finite, we call them finite-product weights of order $\omega$.

Product weights were introduced in [22] and have been studied extensively since then. Finite-product weights were considered in [9]. Observe that for $\sigma \in \mathbb{N}$ the cut-off weights $\gamma^{(\sigma)}$ of product weights $\gamma$ are finite-product weights of order $\sigma$.

Let us assume that $\gamma$ are product or finite-product weights. As shown in [9. Lemma 3.8], we have

$$
\operatorname{decay}_{\gamma, 1}=\text { decay }_{\gamma, \sigma} \text { for all } \sigma \in \mathbb{N} \cup\{\infty\} .
$$

(Actually, [9, Lemma 3.8] states inequality (19) only for all $\sigma \in \mathbb{N}$. But the proof provided in [9] is also valid for the case $\sigma=\infty$.) In particular, we see that for strong polynomial tractability with respect to the nested subspace sampling model and the class $\mathscr{A}^{\text {ran }}$ or with respect to the unrestricted subspace sampling model and the class $\mathscr{A}^{\text {res }}$ it is necessary that decay $\gamma=\operatorname{decay}_{\gamma, \infty}>1$. Since $t_{1}^{*}=1$, we obtain from Theorem 1, 2 and 3 


$$
p_{\text {nest }}^{\text {ran }} \geq \max \left\{\frac{2}{\alpha}, \frac{2 s}{\text { decay }_{\gamma, 1}-1}\right\},
$$

and

$$
p_{\text {unr }}^{\mathrm{res}} \geq \max \left\{\frac{2}{\alpha}, \frac{2 \min \{1, s\}}{\text { decay }_{\gamma, 1}-1}\right\}, \quad p_{\mathrm{unr}}^{\mathrm{res}-\omega} \geq \max \left\{\frac{2}{\alpha}, \frac{2}{\text { decay }_{\gamma, 1}-1}\right\} .
$$

Note that the bounds for finite-product weights are the same as for product weights.

Remark 1. For product and and order-dependent $(P O D)$ weights $\left(\gamma_{u}\right)_{u \in \mathscr{U}}$, which were recently introduced in [15] and are of the form

$$
\gamma_{u}=\Gamma_{|u|} \prod_{j \in u} \gamma_{j}, \quad \text { where } \gamma_{1} \geq \gamma_{2} \geq \cdots \geq 0 \text {, and } \Gamma_{0}=\Gamma_{1}=1, \Gamma_{2}, \Gamma_{3}, \ldots \geq 0,
$$

identity (19) still holds; for a proof see [6]. Thus (20) and (21) are also valid for POD weights. Product and finite-product weights are, in particular, POD weights.

Let us assume that there exist constants $c, C, \kappa>0, \alpha_{1} \geq 0$, and $\alpha_{2} \in[0,1]$ such that for all $\emptyset \neq u \in \mathscr{U}$ and all $n \geq 1$ there exist randomized algorithms $Q_{n, u}$ using for all $f_{u} \in H_{u}$ at most $n$ function values of $f_{u}$ with

$$
\mathbb{E}\left(\left|I_{u}\left(f_{u}\right)-Q_{n, u}\left(f_{u}\right)\right|^{2}\right) \leq \frac{c C^{|u|}}{(n+1)^{\kappa}}\left(1+\frac{\ln (n+1)}{(|u|-1)^{\alpha_{2}}}\right)^{\alpha_{1}(|u|-1)^{\alpha_{2}}}\left\|f_{u}\right\|_{H_{u}}^{2} .
$$

Note that necessarily $\kappa \leq \alpha$. Let us further assume that decay ${ }_{\gamma, 1}>1$ and the cost function $\$$ satisfies $\$(v)=O\left(e^{r v}\right)$ for some $r \geq 0$. Plaskota and Wasilkowski proved in [21] with the help randomized changing dimension algorithms that

$$
p_{\mathrm{unr}}^{\mathrm{res}} \leq \max \left\{\frac{2}{\kappa}, \frac{2}{\text { decay }_{\gamma, 1}-1}\right\} .
$$

Hence, if $\Omega(v)=\$(v)=O\left(e^{r v}\right)$ and $\kappa=\alpha$, our lower bound (21) is sharp and the randomized algorithms from [21] exhibit essentially the optimal convergence rate.

Let us consider a more specific example, namely the case where $D=[0,1], k$ is the Wiener kernel given by $k(x, y)=\min \{x, y\}$, and $\rho$ is the restriction of the Lebesgue measure to $D$. In this case the anchor $a$ is zero. The space $H(k)$ is the Sobolev space anchored at zero, and its elements are the absolutely continuous functions $f$ with $f(0)=0$ and square-integrable first weak derivative. It is known that $\kappa=3=\alpha$, see [26, Ex. 1 and Prop. 3] (or [21, Ex. 2]) and [19, Sect. 2.2.9, Prop. 1]. Thus the upper bound from [21] and our lower bound (21) establish for $\Omega(v)=\$(v)=O\left(e^{r v}\right)$ that

$$
p_{\text {unr }}^{\text {res }}=\max \left\{\frac{2}{3}, \frac{2}{\text { decay }_{\gamma, 1}-1}\right\} .
$$


For the same specific example Hickernell et al. showed for the case $\$(v)=\Theta(v)$ with the help of multilevel Monte Carlo algorithms that

$$
p_{\text {nest }}^{\text {ran }} \leq \max \left\{2, \frac{2}{\text { decay }_{\gamma, 1}-1}\right\} \quad \text { for decay }_{\gamma, 1}>1,
$$

see [13, Cor. 5]. Hence

$$
p_{\text {nest }}^{\text {ran }}=\frac{2}{\text { decay }_{\gamma, 1}-1} \quad \text { for } \text { decay }_{\gamma, 1} \in(1,2] .
$$

Similarly as in the deterministic setting [18, 6] or in the randomized setting with underlying ANOVA-type decomposition [4], our lower bound for $p_{\text {nest }}^{\text {ran }}$ is sharp for sufficiently large decay ${ }_{\gamma, 1}$. This may be proved by using multilevel algorithms based on the integration algorithms provided in [26, Sect. 4] (cf. also [21, Sect. 3.2]) or on scrambled scrambled quasi-Monte Carlo algorithms similar to those discussed in [3], but providing a rigorous proof for this claim is beyond the scope of this article.

\subsubsection{Finite-Intersection Weights}

We restate Definition 3.5 from [9].

Definition 4. Let $\rho \in \mathbb{N}$. Finite-order weights $\left(\gamma_{u}\right)_{u \in \mathscr{U}}$ are called finite-intersection weights with intersection degree at most $\rho$ if we have

$$
\left|\left\{v \in \mathscr{U} \mid \gamma_{v}>0, u \cap v \neq \emptyset\right\}\right| \leq 1+\rho \text { for all } u \in \mathscr{U} \text { with } \gamma_{u}>0 .
$$

For finite-intersection weights of order $\omega$ it was observed in [9] that $t_{\sigma}^{*}=1$ for all $\sigma \in \mathbb{N}$, resulting in the lower bounds (20) and (21) with decay ${ }_{\gamma, 1}$ replaced by $\operatorname{decay}_{\gamma, \omega}$.

\subsubsection{Lexicographically-ordered weights}

To every set $u \subset \mathbb{N}$ with $|u|=\ell$ we may assign a word $\varphi(u):=i_{1} i_{2} \ldots i_{\ell}$, where for $j \in[\ell]$ the number $i_{j}$ is the $j$ th-largest element of $u$. On the set of all finite words over the alphabet $\mathbb{N}$ we have the natural lexicographical order $\prec_{\text {lex }}$, where by convention the empty word should be the first (or "smallest") word.

Definition 5. We call weights $\gamma$ lexicographically-ordered weights of order $\omega$ if $\gamma_{\emptyset}=1, \gamma_{u}>0$ for all $u \subset \mathbb{N}$ with $|u| \leq \omega$, and

$$
\varphi\left(u_{i}\right) \prec_{\operatorname{lex}} \varphi\left(u_{j}\right) \quad \text { for all } i, j \in \mathbb{N} \text { satisfying } i<j .
$$

Lexicographically-ordered weights were introduced in [9]. Their properties complement the properties of the other classes of weights considered before, see [6, 9] 
for more information. For lexicographically-ordered weights of order $\omega$ we have $t_{\sigma}^{*}=\min \{\sigma, \omega\}$. Hence we get from Theorem 1, 2, and 3 the lower bounds

$$
p_{\text {nest }}^{\text {ran }} \geq \max \left\{\frac{2}{\alpha}, \frac{2 s / \omega}{\text { decay }_{\gamma, \omega}-1}\right\},
$$

and

$$
p_{\text {unr }}^{\text {res }} \geq \max \left\{\frac{2}{\alpha}, \frac{2 \min \{1, s / \omega\}}{\operatorname{decay}_{\gamma, \omega}-1}\right\}, \quad p_{\text {unr }}^{\text {res }-\omega} \geq \max \left\{\frac{2}{\alpha}, \frac{2}{\operatorname{decay}_{\gamma, \omega}-1}\right\} .
$$

The lower bounds indicate that in the setting where $\omega>s$ and decay $\gamma_{\gamma, \omega}$ is only moderate, changing dimension algorithms (which are algorithms of the class $\mathscr{A}^{\text {res- }} \omega$ ) cannot achieve the optimal rate of convergence and can be outperformed by multilevel algorithms (which can exhibit a behavior similar to the lower bound for $p_{\text {nest }}^{\text {ran }}$ above). For the deterministic setting and the Wiener kernel $k(x, y)=\min \{x, y\}$ on $D=[0,1]$ this was rigorously proved in [9] by lower bounds for changing dimension algorithms and upper bounds for multilevel algorithms, see [9. Thm. $3.2 \&$ Sect. 3.2.3 ].

\section{Acknowledgment}

The author gratefully acknowledges support by the German Science Foundation (DFG) under grant GN 91/3-1 and by the Australian Research Council (ARC).

\section{References}

1. Aronszajn, N.: Theory of reproducing kernels. Trans. Amer. Math. Soc. 68, 337-404 (1950)

2. Baldeaux, J.: Scrambled polynomial lattice rules for infinite-dimensional integration. In: Plaskota, L., Woźniakowski, H. (eds.) Monte Carlo and Quasi-Monte Carlo Methods 2010. Springer, Heidelberg (2012)

3. Baldeaux, J., Dick, J.: A construction of polynomial lattice rules with small gain coefficients. Numer. Math. 119, 271-297 (2011)

4. Baldeaux, J., Gnewuch, M.: Randomized multilevel algorithms for infinite-dimensional integration on function spaces with ANOVA-type decomposition. Preprint (2012)

5. Creutzig, J., Dereich, S., Müller-Gronbach, T., Ritter, K.: Infinite-dimensional quadrature and approximation of distributions. Found. Comput. Math. 9, 391-429 (2009)

6. Dick, J., Gnewuch, M.: Infinite-dimensional integration in weighted Hilbert spaces: anchored decompositions, deterministic algorithms, and higher order convergence. Preprint (2012)

7. Dick, J., Sloan, I. H., Wang, X., Woźniakowski, H.: Good lattice rules in weighted Korobov spaces with general weights. Numer. Math. 103, 63-97 (2006)

8. Giles, M. B.: Multilevel Monte Carlo path simulation. Oper. Res. 56, 607-617 (2008)

9. Gnewuch, M.: Infinite-dimensional integration on weighted Hilbert spaces. Math. Comp. 81 , 2175-2205 (2012) 
10. Gnewuch, M., Mayer, S., Ritter, K.: On an orthogonal decomposition in weighted Hilbert spaces of functions with infinitely many variables. In preparation.

11. Griebel, M., Holtz, M.: Dimension-wise integration of high-dimensional functions with applications to finance. J. Complexity 26, 455-489 (2010)

12. Heinrich, S.: Monte Carlo complexity of global solution of integral equations. J. Complexity 14, 151-175 (1998)

13. Hickernell, F.J., Müller-Gronbach, T., Niu, B., Ritter, K.: Multi-level Monte Carlo algorithms for infinite-dimensional integration on $\mathbb{R}^{\mathbb{N}}$. J. Complexity 26, 229-254, (2010)

14. Hickernell, F.J., Wang, X.: The error bounds and tractability of quasi-Monte Carlo algorithms in infinite dimension. Math. Comp. 71, 1641-1661 (2001)

15. Kuo, F. Y., Schwab, C., Sloan, I. H.: Quasi-Monte Carlo finite element methods for a class of elliptic partial differential equations with random coefficients. Preprint (2011)

16. Kuo, F. Y., Sloan, I. H., Wasilkowski, G. W., Woźniakowski, H.: Liberating the dimension. J. Complexity 26, 422-454 (2010)

17. Kuo, F. Y., Sloan, I. H., Wasilkowski, G. W., Woźniakowski, H.: On decompositions of multivariate functions. Math. Comp. 79, 953-966 (2010)

18. Niu, B., Hickernell, F. J., Müller-Gronbach, T., Ritter, K.: Deterministic multi-level algorithms for infinite-dimensional integration on $\mathbb{R}^{\mathbb{N}}$. J. Complexity 27, 331-351 (2011)

19. Novak, E..: Deterministic and Stochastic Error Bounds in Numerical Analysis. Lect. Notes in Math. 1349. Springer-Verlag, Berlin (1988)

20. Novak, E., Woźniakowski, H.: Tractability of Multivariate Problems, Volume II. European Mathematical Society, Zürich (2010)

21. Plaskota, L., Wasilkowski, G. W.: Tractability of infinite-dimensional integration in the worst case and randomized setting. J. Complexity 27, 505-518 (2011)

22. Sloan, I. H., Woźniakowski, H.: When are quasi-Monte Carlo algorithms efficient for high dimensional integrals? J. Complexity 14, 1-33 (1998)

23. Traub, J. F., Wasilkowski, G. W., Woźniakowski, H.: Information-Based Complexity. Academic Press, New York (1988)

24. Wasilkowski, G. W.: Randomization for continuous problems. J. Complexity 5, 195-218 (1989)

25. Wasilkowski, G. W., Woźniakowski, H.: On tractability of path integration. J. Math. Physics 37, 2071-2088 (1996)

26. Wasilkowski, G. W., Woźniakowski, H.: The power of standard information for multivariate approximation in the randomized setting. Math. Comp. 76, 965-988 (2007) 